\title{
MODELO POISSON NA DISTRIBUIÇÃO DE CHEGADAS DE CLIENTES
}

Lilian Mari Kieling Ries

Departamento de Matemática. Centro de Ciências Naturais e Exatas.

UFSM. Santa Maria, RS.

Ricardo Iserhardt Ries

Departamento de Engenharia Industrial. Centro de Tecnologia. UFSM. Santa Maria, RS.

\section{RESUMO}

0 artigo analisa a distribuição de chegadas de clientes a um supermercado e utiliza o teste de aderência Qui-Quadrado para ve rificar se as mesmas se ajustam ao modelo poisson.

SUMMARY

RIES,L.M.K. and RIES,R.I., 1979. Poisson Model in the distribution of Client Arrivals. Ciência e Natura (1): 31-38.

The article analyses the distribution of client arrivals to a supermarket. The adherence Q-Square test is used to check if the arrivals agrees with the Poisson model.

INTRODUÇAOO

0 modelo de distribuição de chegadas dos clientes, num sis tema de filas de espera, é um fator importante e deve ser analisado com bastante rigorismo.

Segundo a bibliografia especializada, o modelo de distri buição de chegadas de clientes que se verifica com mais freqüencia segue uma distribuição segundo o modelo Poisson.

Em razão disto, para analisar o processo de chegadas de clientes a um supermercado partiu-se da premissa que estas ocorre riam segundo o modelo Poisson. Para testar o ajustamento dos dados coletados ao modelo Poisson foi utilizado o teste Qui-Quadrado, de aderência.

MODELO POISSON DE CHEGADAS

Considere-se $\alpha$, o intervalo de tempo médio entre duas che gadas consecutivas e $\lambda$ a taxa média de chegadas na unidade de tempo.

Portanto:

$$
\lambda=\frac{1}{\alpha}
$$

Como os clientes não chegam simultaneamente e sim um por vez, a probabilidade de chegada de um cliente, em um intevalo de tem po dt, após a chegada de um anterior, será diretamente proporcional 
a dt e inversamente proporcional a $\alpha$.

Logo:

$$
P_{1}(d t)=\frac{d t}{\alpha}
$$

ou

$$
P_{1}(d t)=\lambda d t
$$

A probabilidade de não chegar nenhum cliente no intervalo de tempo dt, serā:

$$
\begin{aligned}
& P_{0}(d t)=1-P_{1}(d t) \\
& P_{0}(d t)=1-\lambda d t
\end{aligned}
$$

A probabilidade de terem chegado $n$ clientes no período $t$ e nenhum no intervalo dt será dada por:

$$
P_{n}(t) \cdot(1-\lambda d t)
$$

A probabilidade de terem chegado $n-1$ elementos no perīo do $t$ e um intervalo dt serā:

$$
P_{n-1}(t) \cdot \lambda d t
$$

A soma das probabilidades dadas por (1) e (2) nos fornece a probabilidade de haverem chegado $n$ elementos no intervalo $(t+d t)$. Portanto:

$$
P_{n}(t+d t)=P_{n}(t)(1-\lambda d t)+P_{n-1}(t) \cdot \lambda d t \quad \text { para } n \geq 1
$$

ou

$$
P_{n}(t+d t)-P_{n}(t)=-\lambda P_{n}(t)+\lambda P_{n-1}(t)
$$

Levando ao limite com dt $\rightarrow 0$, teremos:

$P_{n}^{\prime}(t)=-\lambda P_{n}(t)+\lambda P_{n-1}(t), \operatorname{com} n \geq 1$

A probabilidade de não chegar nenhum cliente no intervalo $t+d t$ serā correspondente ao produto da probabilidade de não haver chegado nenhum elemento no período $t$ pela probabilidade de não haver chegado nenhum elemento no período dt. Logo:

ou

$$
P_{0}(t+d t)=P_{0}(t) \cdot(1-\lambda d t)
$$

$$
\begin{aligned}
& P_{0}(t+d t)-P_{0}(t)=-\lambda P_{0}(t) d t \\
& \frac{P_{0}(t+d t)-P_{0}(t)}{d t}=-\lambda P_{0}(t)
\end{aligned}
$$

Levando ao limite com dt $\rightarrow 0$, teremos:

$$
P_{j}^{\prime}(t)=-\lambda P_{0}(t)
$$

Segundo NOVAES (1), atravēs do cālculo diferencial e da transformada de CARSON-LAPLACE, as expressões (3) e (4) fornecem as probabilidades: 


$$
\begin{aligned}
& P_{0}(t)=e^{-\lambda t} \\
& P_{1}(t)=\lambda t e^{-\lambda t} \\
& P_{2}(t)=\frac{(\lambda t)^{2} e^{-\lambda t}}{2} \\
& \vdots \\
& P_{n}(t)=\frac{(\lambda t)^{n} e^{-\lambda t}}{n !} \text { com } n=0,1,2 \text { e } \lambda>0 \\
& \text { Esta expressão fornece a probabilidade de haverem chega }
\end{aligned}
$$
do $n$ elementos no intervalo $t+d t$; representa uma distribuição de Poisson, com média $\lambda t$ e variância $\lambda t$.

DETERMINAÇAOO DAS CHEGADAS DE CLIENTES

Para verificar o mecanismo das chegadas, a coleta de da dos foi efetuada, num supermercado, durante 149 dias, em períodos aleatōrios. A unidade de tempo adotada foi de 15 minutos. Assim, foi determinado o nūmero médio de clientes que chegaram ao supermercado em cada 15 minutos. Estes dados se encontram na Tabela I de Freqüẹn cia.

TABELA I. MECANISMO DE CHEGADAS DE CLIENTES AO SUPERMERCADO

\begin{tabular}{cccc}
\hline \hline CHEGADAS & FREQUENCIA & CHEGADAS & FREQUENCIA \\
\hline 15 & 0 & 41 & 9 \\
16 & 1 & 42 & 6 \\
17 & 0 & 43 & 7 \\
18 & 1 & 44 & 4 \\
19 & 1 & 45 & 4 \\
20 & 2 & 46 & 3 \\
21 & 0 & 47 & 4 \\
22 & 3 & 48 & 2 \\
23 & 1 & 49 & 1 \\
24 & 2 & 50 & 2 \\
25 & 1 & 51 & 1 \\
26 & 2 & 52 & 1 \\
27 & 1 & 53 & 1 \\
28 & 2 & 54 & 2 \\
29 & 3 & 55 & 1 \\
30 & 2 & 56 & 1 \\
31 & 4 & 57 & 1 \\
32 & 3 & 58 & 2 \\
33 & 5 & 59 & 1 \\
34 & 60 & 0 \\
35 & 6 & 61 & 1 \\
36 & 7 & 62 & 2 \\
37 & 6 & 63 & 1 \\
38 & 8 & 64 & 149 \\
39 & 8 & 65 & $\mathrm{~T}$ \\
40 & 8 & 0 & 1 \\
\hline
\end{tabular}

Supondo que as chegadas dos clientes ao supermercado obe deçam ao processo poisson, foi elaborado um programa para computador, conforme diagrama da Figura 1, a seguir, para verificar a ajustagem dos dados coletados. O teste de aderēncia aplicado foi do Qui-Quadra 
do de PEARSON. A listagem do programa e os resultados se encontram no Anexo I. Estes resultados permitem admitir que as chegadas dos clientes ao supermercado se ajustam razoavelmente a uma distribuição de Poisson com média de 39,5570 clientes por minutos.

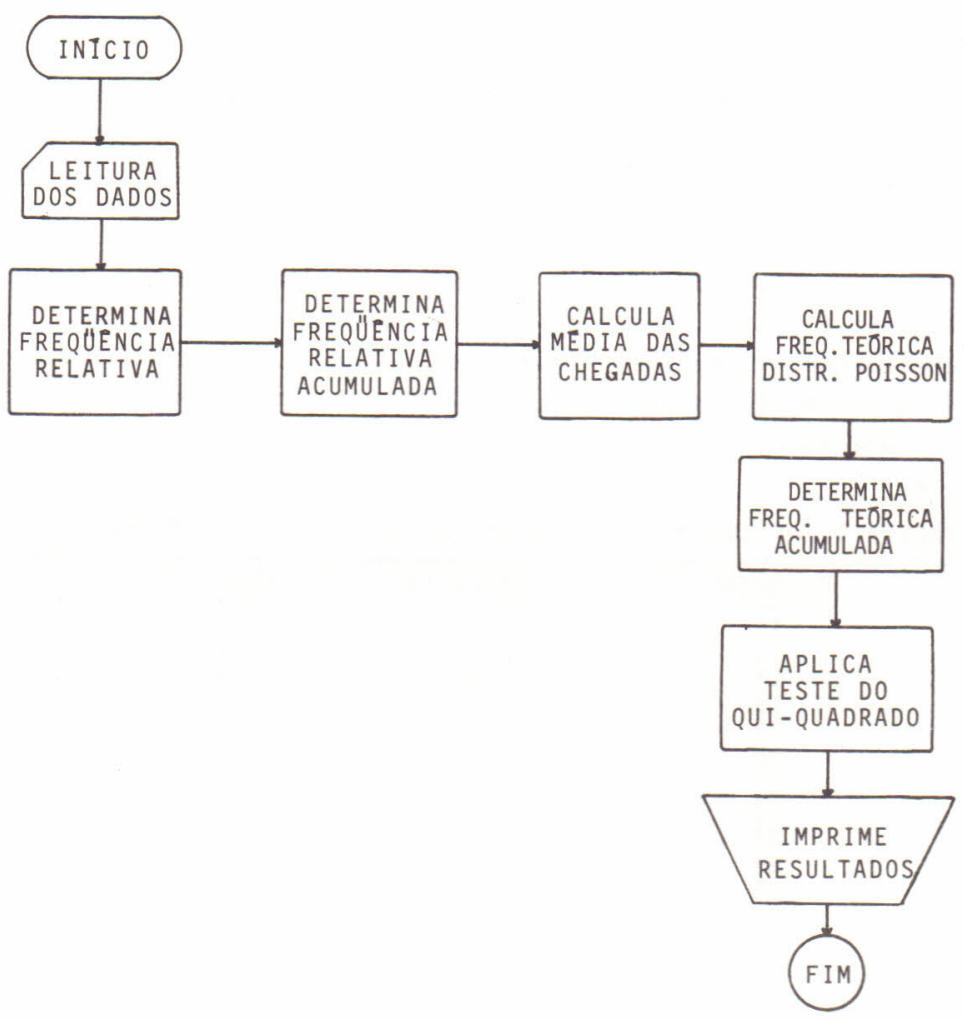

Figura 1. Anālise da distribuição das chegadas de clientes.

O teste do Qui-Quadrado é dado por:

$$
x_{c}^{2}=\frac{\left(o_{j}-e_{j}\right)^{2}}{e_{j}}
$$

onde, $0_{j}$ são as freqüências relativas observadas e

$e_{j}$ são as freqüências relativas teōricas. O resultado forneci do pelo teste foi: $x^{2}=17,0918$.

Como, para 50 graus de liberdade, ao nỉvel de significān cia 0,05 , temos $x_{0,95}^{2}=67,5$, aceitamos a hipótese de que os dados observados seguem uma distribuição de Poisson. 
Na Figura 2 foram representadas as freqüências observadas e as freqüencias teōricas da distribuição de chegadas dos clientes ao supermercado. A figura 3 mostra as freqüencias relativas acumula das, observadas e teōricas. Pode-se verificar, atravēs destes gräf cos, que a aproximação dos dados observados e teōricos ē bem razoā vel.

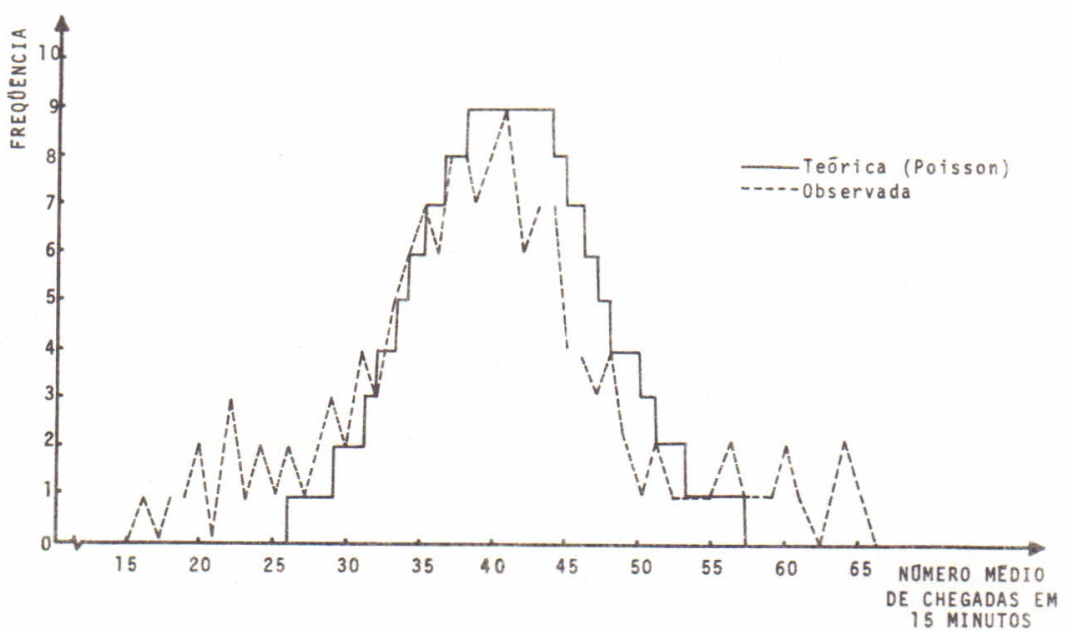

Figura 2. Distribuição das chegadas dos clientes ao supermercado.

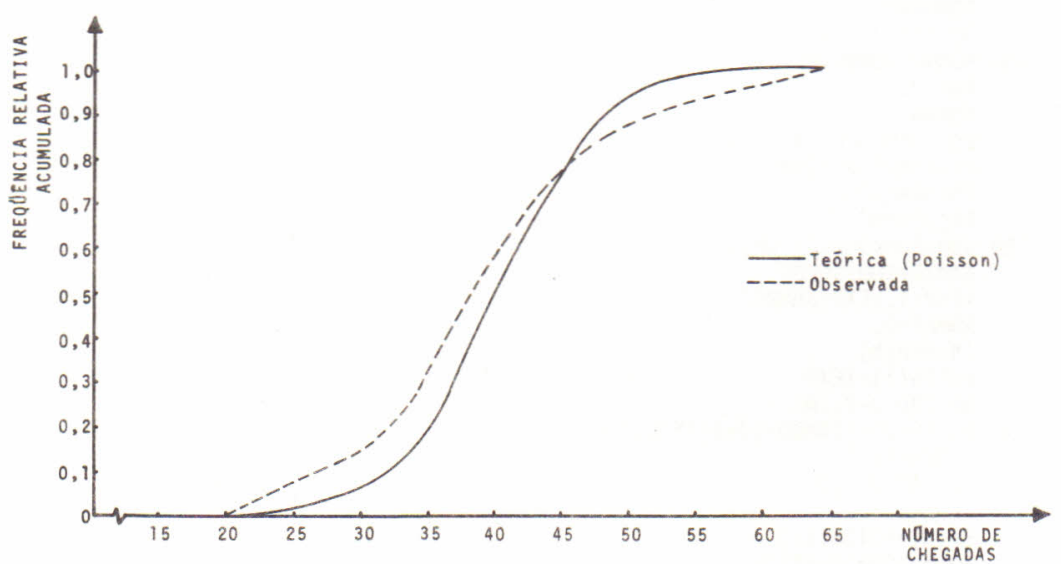

Figura 3. Distribuição cumulativa das chegadas dos clientes ao supermercado. CONCLUSAOO

Considerando-se que o objetivo desta pesquisa era verifi car se a distribuição de chegadas de clientes a um supermercado ocorre 
riam segundo o modelo Poisson, conclui-se, atravēs de anālise esta tística, que as mesmas ocorrem segundo esta distribuição.

0 modelo de computador para testar a ajustagem de dados a uma distribuição de Poisson foi construido de forma generalizada e pode ser utilizado para outras distribuições de chegadas.

BIBLIOGRAFIA CITADA

1. NOVAES,A.G. Pesquisa operacional e transportes: modelos probabi Listicos. São Paulo, Mc Graw-Hill do Brasil, 1975. 293 p.

Recebido em outubro, 1979; aceito em outubro, 1979.

\section{ANEXO I}

C ANALISE ESTATISTICA DAS CHEGADAS

C DISTRIBUICAO DE POISSON

C

C

AMBD $=$ NUMERO MEDIO TOTAL DE CHEGADAS OBSERVADAS

$\mathrm{NCH}(\mathrm{J})=$ NUMERO MEDIO DE CHEGADAS P/15 MIN.

$\operatorname{NP}(\mathrm{J})=$ FREQUENCIA OBSERVADA DE NCH(J)

DIMENSION NCH $(100), \operatorname{NP}(100), P A(100), P(100), P P(100), \operatorname{SOMPP}(100), \operatorname{NFE}(1$

100)

DIMENSION XNFE(100), DIF (100), POISS (100)

$J=1$

$3 \operatorname{READ}(8,100) N C H(J), N P(J)$

100 FORMAT(2I3)

$\operatorname{IF}(\mathrm{NCH}(\mathrm{J})) 4,5,5$

$5 \mathrm{~J}=\mathrm{J}+1$

GOTO 3

$4 \mathrm{~N}=\mathrm{J}-1$ SOMNP $=0$.

DO $110 \mathrm{~J}=1, \mathrm{~N}$

100 SOMNP $=$ SOMNP+NP(J)

$\mathrm{PAC}=0$.

$\mathrm{SOM}=0$.

DO $120 \mathrm{~J}=1, \mathrm{~N}$

$P(J)=N P(J) / S O M N P$

$P A C=P A C+P(J)$

$P A(J)=P A C$

$120 \mathrm{SOM}=\mathrm{SOM}+\mathrm{NCH}(\mathrm{J}) * \mathrm{NP}(\mathrm{J})$

XAMBD $=$ SOM $/$ SOMNP

XEXP=1./EXP (XAMBD)

SOMAT $=0$.

$\mathrm{IN}=\mathrm{NCH}(\mathrm{N})$

$\operatorname{POISS}(1)=\operatorname{XEXP}$

DO $230 \mathrm{~J}=2$, IN

$230 \operatorname{POISS}(\mathrm{J})=(\operatorname{XaMBD} /(\mathrm{J}-1)) * \operatorname{POISS}(\mathrm{J}-1)$

$\mathrm{L}=\mathrm{NCH}(1)-1$

DO $140 \mathrm{~J}=1, \mathrm{~N}$

$\mathrm{LL}=\mathrm{J}+\mathrm{L}$

$\operatorname{PP}(J)=P O I S S(L L)$

SOMAT $=$ SOMAT $+P P(\mathrm{~J})$

$140 \operatorname{SOMPP}(\mathrm{J})=$ SOMAT

WRITE $(5,150)$ XAMBD, N

150 FORMAT (1 H $1, / / /, 15 \mathrm{X}$, 'ESTUDO ESTATISTICO DAS CHEGADAS', //,15X, 'NUMER

10 MEDIO DE CHEGADAS POR 15 MIN. =' $, F 10.4, / /, 15 X$, 'NUMERO DE DADOS CO

2 LETADOS $=1$, I 5 )

$\operatorname{WRITE}(5,90)$ 
90 FORMAT(//, 10X, 'NUM.DE ',3X'FREQ. ' ,3X, 'FREQ. ', 3X, 'FREQ. ' ,3X, 'FREQ.

1 ',3X, 'FREQ. TEOR.' ',/, 10X, 'CHEGADAS', 3X, 'OBSERV' ,3X, 'RELAT' , 3X, 'ACU

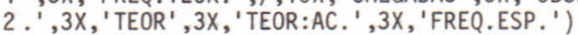

SOMDF $=0$.

DO $170 \mathrm{~J}=1, \mathrm{~N}$

$\operatorname{NFE}(J)=(P P(J) * S O M N P+0.5)$

$\operatorname{XNFE}(\mathrm{J})=(P P(\mathrm{~J}) \star \mathrm{SOMNF}+0.5)$

$\operatorname{DIF}(\mathrm{J})=\left((P(\mathrm{~J})-\mathrm{PP}(\mathrm{J}))^{\star \star 2}\right) / P P(\mathrm{~J})$

170 SOMDF $=$ SOMDF+DIF $(\mathrm{J})$

WRITE $(5,160)(N C H(J), N P(J), P(J), P A(J), P P(J), \operatorname{SOMPP}(J), N F E(J), J=1, N)$

160 FORMAT $(12 X, 14,5 X, 13,4 F 9.3,3 X, I 4)$

WRITE $(5,300)$ SOMDF

300 FORMAT $\left(/ /, 10 X, X^{\star \star} 2=', F 10.4\right)$

CALL EXIT

END

ESTUDO ESTATISTICO DAS CHEGADAS

NUMERO MEDIO DE CHEGADAS POR $15 \mathrm{MIN} .=39.5570$

NUMERO DE DADOS COLETADOS - 51

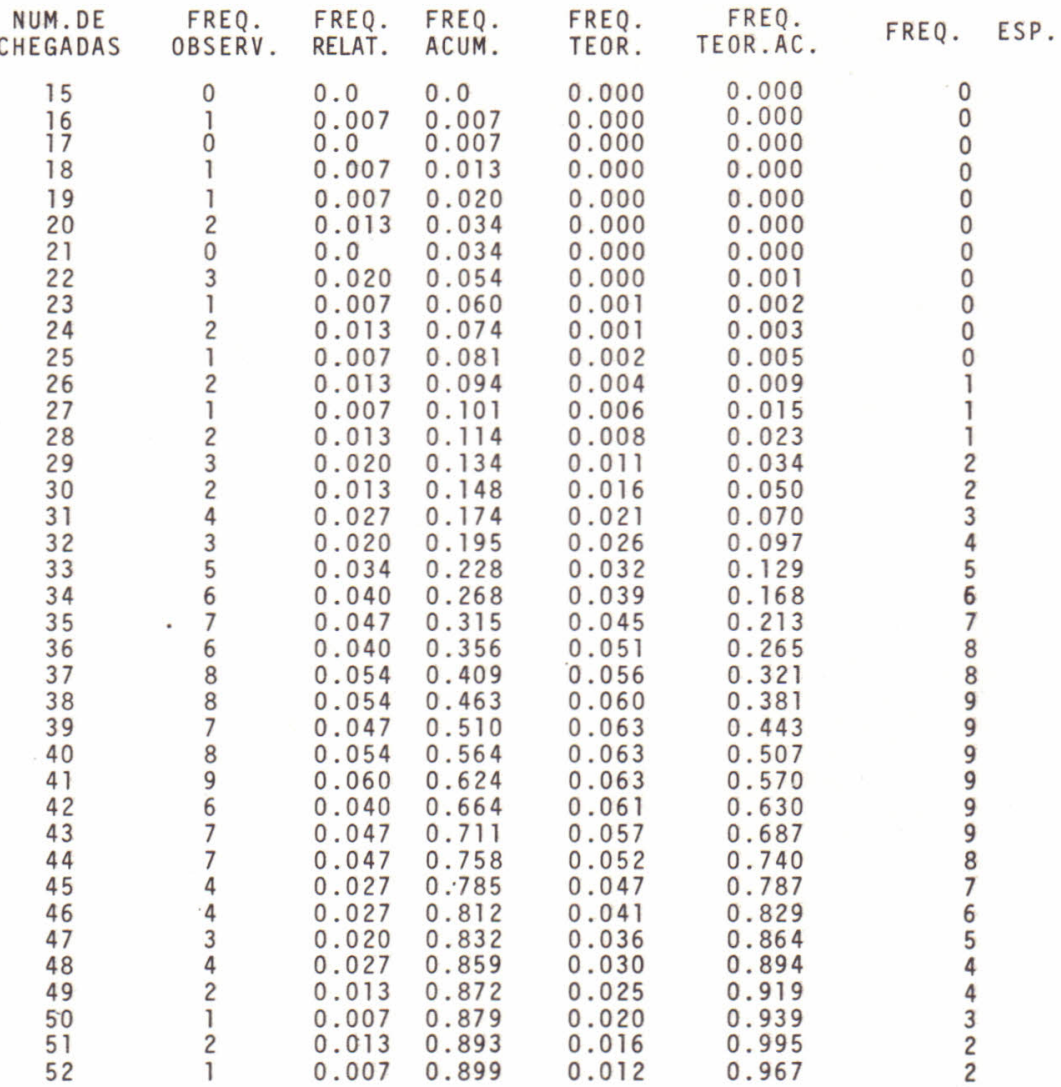




$\begin{array}{lllllll}53 & 1 & 0.007 & 0.906 & 0.009 & 0.976 & 1 \\ 54 & 1 & 0.007 & 0.913 & 0.007 & 0.983 & 1 \\ 55 & 1 & 0.007 & 0.919 & 0.005 & 0.988 & 1 \\ 56 & 2 & 0.013 & 0.933 & 0.004 & 0.992 & 1 \\ 57 & 1 & 0.007 & 0.940 & 0.003 & 0.995 & 0 \\ 58 & 1 & 0.007 & 0.946 & 0.002 & 0.996 & 0 \\ 59 & 1 & 0.007 & 0.953 & 0.001 & 0.998 & 0 \\ 60 & 2 & 0.013 & 0.966 & 0.001 & 0.999 & 0 \\ 61 & 1 & 0.007 & 0.973 & 0.001 & 0.999 & 0 \\ 62 & 0 & 0.0 & 0.973 & 0.000 & 0.999 & 0 \\ 63 & 1 & 0.007 & 0.980 & 0.000 & 1.000 & 0 \\ 64 & 2 & 0.013 & 0.993 & 0.000 & 1.000 & 0 \\ 65 & 1 & 0.007 & 1.000 & 0.000 & 1.000 & \end{array}$

$x^{\star \star 2}=17.0918$ 